\section{Miomas e infertilidade: bases fisiopatológicas e implicações terapêuticas}

\section{Uterine leiomyomas and infertility: physiopathological basis and therapeutical implications}

Ana Luiza Berwanger da Silva 1

Samuar Albano Seibel 2

Edison Capp 3

Helena von Eye Corleta 4

1-4 Departamento de Ginecologia e Obstetrícia.

Faculdade de Medicina da Universidade Federal do Rio Grande do Sul. Rua Ramiro Barcelos, 2400, $4^{\circ}$ andar. Porto Alegre, RS, Brasil. CEP: 90.035-003

\begin{abstract}
Objectives: to analyze evidences in medical literature concerning the possible relation between uterine leiomyomas and infertility, and the advantages /disadvantages of the different treatments available.

Methods: literature review using the key words (MeSh terms) "leiomyomas", "infertility", "treatment", "myomectomy", "pregnancy" in "Pubmed" and "The Cochrane Library 2003" database. The search period was from 1989 through 2003.

Results: although there are contradictory results, the majority of authors determined a possible cause-consequence relation between some types of leiomyomas and reproductive impairement. Concerning therapeutics, we were able to clearly place myomectomy as being the surgical technique with the best results. Surgical approaches used for this procedure did not show significant differences from each other, except for submucosal myomas, where histeroscopy is more effective. Other existent treatment options do not seem to be indicated for infertile patients with uterine leiomyomas.

Conclusions: the possible association between myomas and reproductive impairment needs to be further investigated. Success indicated by gestational rates following surgical resection, mainly in patients with submucosal myomas, are an indication that they do have a role in infertility etiology. Concerning the treatment, it became clear that myomectomy is the procedure of choice for all patients desiring to become pregnant.
\end{abstract}

Key words Myomas, Infertility, female, Pregnancy

\section{Resumo}

Objetivos: analisar evidências da literatura médica que avaliam a relação entre miomas uterinos e infertilidade, assim como as vantagens e desvantagens dos diferentes tipos de tratamentos disponiveis.

Métodos: foi realizada uma revisão da literatura utilizando os termos (MeSh terms) "miomas", "infertilidade", "tratamento", "miomectomia", "gestação", na base de dados "Pubmed" e na "The Cochrane Library 2003". O período analisado foi de 1989 a 2003.

Resultados: apesar de alguns resultados contraditórios, a maioria dos autores relata uma possivel relação de causa-conseqüência entre determinados tipos de miomas e distúrbios reprodutivos. Em relação à terapêutica, observa-se claramente que a miomectomia representa a técnica cirúrgica com melhores resultados, não havendo diferença significativa entre as vias de acesso utilizadas, com exceção da miomatose submucosa, situação em que a via histeroscópica é preferida. As opções não-cirúrgicas de tratamento existentes não parecem ter indicação em pacientes inférteis.

Conclusões: a possivel associação entre miomas e distúrbios reprodutivos necessita de melhores investigações. O incremento nas taxas de gestação após ressecção cirúrgica de miomas submucosos leva-nos a acreditar que esses tumores tenham papel na etiologia da infertilidade. Em miomas de outras localizações, entretanto, a relação com infertilidade não é tão clara. A miomectomia é o procedimento de escolha para as pacientes que ainda desejam engravidar.

Palavras-chave Mioma, Infertilidade feminina, Gravidez 


\section{Introdução}

Conforme o método utilizado para o diagnóstico de miomatose, sua incidência durante a menacme pode variar de 5,4 a 77\%.1-7 Essa taxa aumenta progressivamente com o decorrer da idade.1,3 São particularmente comuns na população de raça negra, em uma proporção de 9:1, quando comparada à população branca. 1,2,6,8 A miomatose é a indicação mais comum de laparotomia em mulheres não grávidas nos Estados Unidos. 2,4

Miomas uterinos são tumores benignos que se desenvolvem a partir das células musculares lisas do miométrio.1,2 Eles podem ser classificados como intramurais (localizados na camada miometrial, com menos de $50 \%$ de seu volume protruindo na superfície serosa do útero), submucosos (localizados na camada interna do miométrio, com projeção para a cavidade uterina), subserosos (quando possuem mais de $50 \%$ do seu volume projetado na camada serosa do útero) e pediculados (ligados ao útero por um pedículo).1,3

Cerca de $75 \%$ das pacientes com miomatose são assintomáticas. Quando presentes, porém, os principais sinais e sintomas encontrados são menorragia e/ou metrorragia, como também massa pélvica, efeitos compressivos (sintomas urinários e intestinais), dor e infertilidade.1,2,4,7 Acredita-se que a menorragia decorra do aumento da superfície intracavitária, a qual altera a contração dos vasos endometriais durante o período menstrual, levando ao engurgitamento dos vasos tumorais. Já o sangramento intermenstrual provavelmente decorre de congestão, necrose e ulceração do endométrio que circunda o mioma. ${ }^{2}$ Esses sintomas geralmente são associados à localização do tumor, sendo que os intramurais e subserosos tendem a causar sintomas compressivos, dor e distorção anatômica de órgãos adjacentes, enquanto que os submucosos produzem sangramento com mais freqüência. Além disso, observou-se que esses últimos estão mais associados à disfunção reprodutiva.4-6,9,10 A degeneração sarcomatosa, é rara $(0,1 \%$ dos casos $) .1,2$

Há muito tempo tem-se especulado que miomas uterinos diminuem a fertilidade, $2,3,6,11-13$ e que sua remoção aumentaria as taxas de gestação.3,11 De uma forma geral, estima-se que esses tumores estejam associados com disfunção reprodutiva em 5\% a $10 \%$ dos casos. 4,13 Quando todas as outras causas possíveis de infertilidade são excluídas, entretanto, eles podem ser responsáveis por apenas $2 \%$ a $3 \%$ dos casos desse distúrbio. 4,5

A associação entre miomas e infertilidade tem sido largamente reconhecida, principalmente quando se trata de tumores com localização submucosa. 1,5,6,9 Não apenas essa hipótese, como também a forma ideal de tratamento para resolução dos sintomas e prevenção de uma possível disfunção reprodutiva têm sido questões de debate já há algum tempo.

\section{Fisiopatologia}

Miomas são tumores estrogênio-dependentes, podendo atingir um tamanho significativo sob a influência desse hormônio.1,4 Essa observação é fortemente sugerida pelo fato de que eles tendem a sofrer involução após a menopausa, assim como sob efeito do tratamento com agonistas do Gonadotropin Releasing Hormone (GnRH). ${ }^{1}$ Existem evidências de que a progesterona também exerça um papel de estímulo ao crescimento tumoral,1,4 além de outros fatores de crescimento locais. 4

A etiologia e a patogênese dos miomas permanecem como um grande enigma.1,3,5,13 Vários mecanismos pelos quais esses tumores possam causar redução da fertilidade têm sido sugeridos:3,4,5,9,12 a) alteração do contorno endometrial, interferindo na implantação; b) aumento e deformação da cavidade uterina, podendo influir no transporte e no acesso dos espermatozóides; c) alteração da contratilidade uterina, prejudicando a movimentação normal do esperma; d) persistência de sangue ou coágulos intra-uterinos, dificultando a implantação;4,5,9,12 e) distorção ou obstrução do óstio tubário;4,11 f) anormalidades da vascularização uterina; g) inflamação intracavitária crônica.5,6,11,13 Nenhum desses mecanismos, porém, foi comprovado como fator etiológico de infertilidade. 4,6,11

Bernard et al. ${ }^{9}$ ainda chamam a atenção para a possível influência de outros fatores fisiopatológicos não relacionados ao mioma em si, como alterações endometriais, já que observaram alta taxa de abortamento mesmo após ressecção histeroscópica em pacientes com miomas submucosos.

Tem sido difícil identificar com precisão quais tipos de miomas realmente alteram a capacidade reprodutiva, pois, muitas vezes, existem outros possíveis fatores de infertilidade associados.

Uma revisão sistemática da literatura a respeito da influência de miomas e de sua ressecção na taxa de fertilidade demonstrou que as mulheres inférteis com miomas intracavitários têm menores taxas de gestação, implantação e parto após FIV (fertilização in vitro) do que o grupo controle (mulheres inférteis sem miomas) e do que mulheres inférteis com miomas sem componente intracavitário. O tamanho tumoral não influenciou significativamente os resul- 
tados terapêuticos, ${ }^{11}$ conclusão também sugerida por outros autores. 9,10

Na série de Donnez e Jadoul 5 houve diminuição significativa nas taxas de gestação após FIV em pacientes com deformação da cavidade uterina (conseqüência, sobretudo de miomas submucosos), quando comparadas a mulheres sem alteração da cavidade ou com infertilidade não associada a miomas. $\mathrm{Ng}$ e Ho, ${ }^{3}$ assim como Pritts ${ }^{11}$ obtiveram resultados semelhantes. Outros autores, porém, em estudos retrospectivos e com pequena amostra observaram resultados divergentes, demonstrando diminuição da fertilidade em pacientes com miomas intramurais sem distorção da cavidade. ${ }^{9}$

\section{Tratamento dos miomas na infertilidade}

O tratamento de miomas depende dos sintomas provocados e dos objetivos terapêuticos. ${ }^{2}$ Ainda existem muitas controvérsias sobre qual a técnica ideal para o tratamento das pacientes com miomas e infertilidade, tanto em termos de taxas de gestação pós-tratamento, quanto de suas complicações. 1,5,6 No entanto, é consenso que a conduta expectante tende a ser a melhor abordagem para mulheres com miomas assintomáticos.4,14 Várias técnicas vêm sendo desenvolvidas e testadas para o tratamento de miomas uterinos. Abaixo, são descritas as principais opções disponíveis atualmente.

\section{Tratamento hormonal}

Esta abordagem baseia-se no fato de que os miomas tendem a se desenvolver na presença de estímulos estrogênico e progestínico e a diminuir sob a ação de androgênios. ${ }^{2}$ A terapia progestínica, incluindo anticoncepção oral, foi proposta para diminuir o tamanho do útero e promover atrofia endometrial, reduzindo assim, o sangramento. Entretanto, a resposta a esse tipo de tratamento não só se mostra ineficaz, como pode resultar em crescimento tumoral.2,4 Similarmente, mulheres tratadas com androgênios, tais como gestrinona e danazol, por vezes experimentam esse mesmo problema, além de efeitos colaterais significativos. Dessa forma, nenhuma dessas opções têm recomendação atualmente. 4

Ao longo dos últimos dez anos, um grande número de estudos tem avaliado o emprego de análogos do GnRH no tratamento da miomatose. Essa abordagem apoia-se no conhecimento da regulação negativa exercida sobre a secreção hipotalâmica de GnRH, levando ao hipoestrogenismo. 4 No estado de secreção estrogênica diminuída, foi observado redução de até $61 \%$ no volume uterino e de até $35 \%$ no volume tumoral, com melhora dos sintomas. Entretanto, no momento em que é descontinuado o tratamento, os miomas tendem a alcançar seu tamanho inicial ou até se tornarem maiores.2,4 Além disso, observou-se uma perda de osso trabecular de até $6 \%$ após seis meses de tratamento, a qual nem sempre é reversível. ${ }^{2}$

Dessa forma, o uso desses hormônios deve ser considerado apenas para casos em que se deseja um alívio sintomático por um período limitado de tempo, 4 ou em casos pré-cirúrgicos, quando a diminuição tumoral pode facilitar a técnica.2,5 Ribeiro et al.,12 porém, não encontraram diferença no grau de dificuldade cirúrgica para ressecção de tumores diminuídos sob influência dessas drogas. Esses autores inclusive ressaltam que a possibilidade de degeneração tumoral dos miomas, tornando sua extirpação ainda mais difícil.

Outra situação em que essa abordagem terapêutica pode ser benéfica se trata de casos de pacientes anêmicas, devido ao risco cirúrgico de sangramento. Foi demonstrado que o uso de análogos do GnRH, juntamente com a suplementação de ferro, aumenta significativamente os valores de hematócrito e hemoglobina dessas pacientes, devido à melhora dos sintomas de meno-metrorragia. Em mulheres nãoanêmicas, todavia, não se observa tal benefício. 4

\section{Tratamento cirúrgico}

O tratamento cirúrgico definitivo na abordagem de miomas é a histerectomia. 1,2,4,7,12 Essa técnica naturalmente não constitui uma opção no manejo de mulheres com infertilidade e sem prole completa.1,4 Para pacientes inférteis ou sintomáticas que desejam engravidar ou conservar o útero, uma boa opção é a miomectomia. Após esse procedimento, observa-se resolução da menorragia e da anemia em cerca de $80 \%$ dos casos. ${ }^{2}$ As indicações principais desta técnica incluem a presença de sintomas, um rápido crescimento, distorção da cavidade uterina e oclusão tubária em pacientes que desejam engravidar. ${ }^{2}$

Complicações associadas à miomectomia em geral não são secundárias à técnica em si, sendo as principais: a) hemorragia maciça intra-operatória; b) necessidade de histerectomia de urgência; c) alteração do contorno da cavidade uterina; $1 \mathrm{~d}$ ) rotura do útero em gestação subseqüente, devido à cicatriz uterina remanescente.1,6 A principal complicação no que diz respeito à fertilidade, porém, é a formação de aderências. O local da incisão uterina influencia de forma importante nesse desfecho, sendo que incisões na parede posterior podem levar ao aparecimento 
dessas lesões em até $94 \%$ dos casos, enquanto que, quando realizadas na parede anterior, resultam em aderências em 55\%.2,4,8,11

Em um estudo prospectivo avaliando a morbidade e o desfecho reprodutivo em pacientes após miomectomia secundária, Frederick et al. ${ }^{8}$ observaram como principal complicação a formação de aderências abdômino-pélvicas, sendo essas de localização tubária em 59\% das pacientes, e intestinal em 90\% dos casos avaliados. Mesmo assim, constatou-se também que, apesar da presença dessas aderências, $67 \%$ das pacientes apresentavam permeabilidade tubária.

As taxas de gestação a termo pós-miomectomia publicadas variam entre $40 \%$ e 50\%.4,8 Entretanto, até $50 \%$ das pacientes permanecem inférteis nos cinco anos subseqüentes à miomectomia primária.

Um estudo italiano retrospectivo obteve como variáveis significativamente associadas à taxa de concepção pós-miomectomia a idade da paciente no momento da cirurgia, a duração da infertilidade antes do procedimento e a presença de outros fatores menores de infertilidade adicionais. 10

Através da análise dos principais estudos avaliando a relação entre miomas e infertilidade, parece não haver correlação entre as taxas de gestação após tratamento por miomectomia e o número e tamanho dos miomas ressecados. ${ }^{4}$ Além disso, observa-se que cerca de $75 \%$ das pacientes submetidas a esse procedimento conseguem engravidar no período de um ano. $4,5,8$

Em sua metanálise, Pritts et al. ${ }^{11}$ observaram que, quando pacientes com miomas submucosos foram comparadas separadamente, a taxa de gestação aumentou significativamente após miomectomia, em comparação com o grupo controle, constituído por mulheres inférteis sem miomas. Além disso, esses autores também descreveram taxas de parto equivalentes entre os dois grupos.

Não existe consenso sobre a melhor via cirúrgica para a realização da miomectomia.2,4 A laparoscopia vem ganhando espaço nos últimos tempos, com a melhoria da técnica e com o aumento do número de médicos capazes de realizá-la de forma eficaz. $4,10,12$ É observada diminuição da dor e do tempo de recuperação pós-operatórios com o uso dessa via. Alguns autores, porém, postulam que a opção pela laparoscopia resulta em maior taxa de complicações, como a rotura uterina em uma gestação subseqüente, devido à maior dificuldade de sutura do miométrio. 4

Quando se trata de miomas de grande volume, podem ser necessárias técnicas adicionais para sua retirada, como o morcelamento ou a coagulação do tumor.2,7 $\mathrm{Na}$ metanálise realizada por Donnez a
Jadou$^{5}$ observaram-se taxas de gestação entre 16,7\% e 73,1\% após miomectomia laparoscópica e cerca de $9,6 \%$ a $75 \%$ após miomectomia abdominal. Essa diferença não se mostrou estatisticamente significativa. Paralelamente, as porcentagens de partos vaginais e de cesarianas foram equivalentes entre as pacientes dos dois grupos. Dessa forma, os autores não dão preferência a alguma das duas vias em particular, devendo ser preferida aquela em que o médico demonstra maior experiência.

Frederick et al. 8 observaram gestações a termo em $56 \%$ das pacientes submetidas a miomectomia secundária em seu estudo. Os autores ressaltam também que essas mulheres diferenciaram-se daquelas que não obtiveram gestação pela menor idade $(31,8 \pm 2,6$ anos versus $35 \pm 1,8$ anos, respectivamente). No entanto, mostrou-se claramente a menor taxa de fertilidade após miomectomia secundária em relação ao procedimento primário, quando comparada com os números descritos na literatura. Essa é uma informação significativa no momento de planejar o tratamento, já que muitas pacientes possuem miomas múltiplos, os quais, por vezes, não são ressecados em sua totalidade, seja por dificuldade técnica, seja pela sua não visualização através dos métodos diagnósticos utilizados. Outra ressalva importante é que, para as pacientes submetidas à miomectomia secundária, deve-se considerar fortemente a opção de fertilização assistida, em especial para aquelas com idade mais avançada.

Em se tratando de miomas intracavitários sintomáticos, sobretudo aqueles com menos de 50\% de volume intramural, outra boa opção cirúrgica é a ressecção histeroscópica. $2,4,5,7,9,13$ Trata-se de uma via que demonstra vantagens quando executada por profissionais experientes, tais como redução do tempo de hospitalização e do custo e, principalmente, menor morbidade. ${ }^{11,13}$ Não existem muitos dados sobre as taxas de gestação após ressecção histeroscópica na literatura; de uma forma geral, observam-se porcentagens entre 31 e 77\%. ${ }^{13}$ Mesmo para miomas de maior volume, a taxa de complicações tende a ser baixa. ${ }^{11}$ Deve-se ressaltar que essa técnica, no entanto, também não é isenta de possíveis contratempos, sendo os principais sangramento, hiponatremia, acúmulo de volume 2 e perfuração uterina (risco maior para tumorações de localização central).2,13

Donnez e Jadoul 5 em uma metanálise sobre estudos avaliando a associação de miomas e infertilidade, não observaram diferenças significativas no desfecho reprodutivo quando comparada a utilização das vias histeroscópica, laparoscópica e laparotômica. Outros autores também descrevem taxas de ferti- 
lidade similares em pacientes submetidas à miomectomia por laparotomia e laparoscopia. 4

Contrariamente a esses dados, uma metanálise publicada em 2001 sugere que existem muitas razões para evitar a miomectomia em pacientes inférteis com miomas subserosos ou intramurais, devido não somente à possibilidade das várias complicações possíveis citadas anteriormente, mas também ao fato de que não existem dados suficientes demonstrando uma associação clara entre infertilidade e esses tipos de miomas.

Tem sido observado que a recorrência média em dez anos pós-miomectomia varia em torno de $27 \%, 4,8$ podendo chegar a $51 \%$, apesar de essa ser menos freqüente em pacientes que apresentavam mioma único. 2

\section{Embolização da artéria uterina (EAU)}

A embolização de vasos uterinos tem sido utilizada desde o início dos anos 90.4 Essa técnica envolve a introdução e manipulação de um cateter via artéria femoral até as artérias uterinas, seguidas da injeção de uma substância esclerosante, com o objetivo de embolizar o fluxo sangüíneo essencial do mioma. Ela é baseada no fato de que os miomas são particularmente suscetíveis à degeneração, pois seu rápido crescimento exige um aumento proporcional de seu suprimento sangüíneo, o qual nem sempre é suficiente. ${ }^{1}$ Além disso, sabe-se que o miométrio normal rapidamente desenvolve uma nova vascularização através de vasos colaterais. ${ }^{7}$

As taxas de sucesso terapêutico relatadas variam de $87 \%$ a $90 \%$, com redução do volume do mioma dominante em $40 \%$ a $65 \% .2,7$ O alívio do sangramento e da dor pélvica parece ser semelhante àquele proporcionado por histerectomia ou miomectomia. Falha de técnica, que ocorre em aproximadamente $10 \%$ dos casos, em geral é associada a úteros de grandes tamanhos, mioma único maior de $8,5 \mathrm{~cm}$, embolização incompleta, variações anatômicas ou adenomiose coexistente. ${ }^{2}$

Algumas complicações significativas, entretanto, são observadas após o emprego deste tipo de tratamento, 1,4,7 tais como dor intensa no período pósoperatório, febre, cólicas abdominais, infecção, hemorragia, grande necrose tecidual e até mesmo retardo no diagnóstico de um leiomiosarcoma. ${ }^{1,4} \mathrm{~A}$ principal e mais danosa, porém, é a falência ovariana precoce secundária à interferência no suprimento sangüíneo ovariano. Essa complicação é descrita em cerca de $5 \%$ a $14 \%$ dos casos, ocorrendo principalmente em mulheres por volta dos 40 anos.1,2 É possível que essa complicação se deva à emboliza- ção de vasos adjacentes, devido às anastomoses uterino-ovarianas. ${ }^{2}$ Assim, pode-se deduzir que esta não é uma opção terapêutica para pacientes que desejam preservar a fertilidade. ${ }^{2,5}$

\section{Terapia gênica}

Ainda como uma futura opção de tratamento, podese citar a terapia gênica. Essa técnica baseia-se na introdução de vetores (virais ou não-virais) portadores de um "DNA terapêutico" (com alterações induzidas ou sem defeitos, quando esses são os causadores da doença), que será incorporado ao DNA celular da paciente. Esse tipo de tratamento pode possuir caráter corretivo, imunológico ou citotóxico, como no caso de miomas, ocorrendo a destruição das células anormais diretamente através de efeitos tóxicos. ${ }^{2}$

A semelhança dos leiomiócitos com os miócitos e fibroblastos os tornam facilmente transferíveis in vitro, fazendo com que a terapia gênica pareça uma provável futura opção para a abordagem desses tumores. 2

\section{Conclusões}

A associação entre miomas e distúrbios reprodutivos ainda hoje permanece não totalmente esclarecida, como ressalta a maioria dos autores. O sucesso demonstrado pelas taxas de gestação após ressecção cirúrgica, principalmente em pacientes com miomas submucosos, leva-nos a acreditar de fato que esses tumores exerçam um papel na etiologia da infertilidade.

Em relação a miomas de outras localizações, entretanto, nem sempre se observa a mesma relação. Apesar de a maioria dos estudos exibirem resultados satisfatórios em termos de melhora reprodutiva após extirpação desses tumores, os dados da literatura não são sempre concordantes e, muitas vezes, levam até a total contradição. Dessa forma, fica a dúvida a respeito do benefício do tratamento desses tumores com finalidade somente reprodutiva. Obviamente, a presença de sintomas acaba se tornando indicação terapêutica formal, independente da existência de infertilidade.

No que tange às diferentes técnicas terapêuticas, está claro que a miomectomia consiste no procedimento de escolha para mulheres inférteis com miomas que ainda desejam engravidar. A via histeroscópica parece ser a preferencial na miomatose submucosa, sendo que as outras vias cirúrgicas disponíveis não possuem vantagens entre si. A escolha deve ser 
baseada nas particularidades de cada caso, assim como aquela com a qual o médico sentir maior segurança e experiência. Outras opções, como a embolização de vasos uterinos e a terapia gênica ainda não se aplicam para esse objetivo.

\section{Referências}

1. Griffiths A, D'Angelo A, Amso N. Surgical treatment of fibroids for subfertility: protocol for a Cochrane review. (Update software). The Cochrane Library 2003; (2).

2. Aubuchon M, Pinto AB, Williams DB. Treatment of uterine fibroids. Prim Care Update Obstet Gynecol 2002; 9: 231-7.

3. Ng EH, Ho PC. Doppler ultrasound examination of uterine arteries on the day of oocyte retrieval in patients with uterine fibroids undergoing IVF. Hum Reprod 2002; 17: 765-70.

4. American Society for Reproductive Medicine. Myomas and reproductive function. Bull Am Soc Reprod Med 2001; (nov): 1-12.

5. Donnez J, Jadoul P. What are the implications of myomas on fertility? Hum Reprod 2002; 17: 1424-30.

6. Hart R, Khalaf Y, Yeong CT, Seed P, Taylor A, Braude P. A prospective controlled study of the effect of intramural uterine fibroids on the outcome of assisted conception. Hum Reprod 2001; 16: 2411-17.

7. Lumsden MA. Embolization versus myomectomy versus hysteroscopy. Which is best, when? Hum Reprod 2002; 17: 253-9.

8. Frederick J, Hardie M, Reid M, Fletcher H, Wynter S, Frederick C. Operative morbidity and reproductive outcome in secondary myomectomy: a prospective cohort study. Hum Reprod 2002; 17: 2967-71.
9. Bernard G, Darai E, Poncelet C, Benifla JL, Madelenat P. Fertility after hysteroscopic myomectomy: effect of intramural myomas associated. Eur J Obstet Gynecol Reprod Biol 2000; 88: 85-90.

10. Vercellini P, Maddalena S, De Giorgi O, Pesole A, Ferrari L, Crosignani PG. Determinants of reproductive outcome after abdominal myomectomy for infertility. Fert Steril 1999; 72: 109-13.

11. Pritts EA. Fibroids and infertility: a systematic review of the evidence. Obstet Gynecol Surv, 2001, 56 (8): 483-91.

12. Ribeiro SC, Reich H, Rosenberg J, Guglielminetti E, Vidali A. Laparoscopic myomectomy and pregnancy outcome in infertile patients. Fertil Steril 1999; 71: 571-4.

13. Varasteh NN, Neuwirth RS, Levin B, Keltz MD. Pregnancy rates after hysteroscopic polypectomy and myomectomy in infertile women. Obstet Gynecol 1999; 94: 169-71.

14. Burton CA, Grimes DA, March CM. Surgical management of leiomyomata during pregnancy. Obstet Gynecol 1989; 74: 707-9.

Apresentado em 11 de março de 2004

Versão final apresentada em 11 de agosto 2004

Aprovado em 17 de setembro de 2004 